\title{
AVALIAÇÃO DO RISCO DE INTOXICAÇÕES POR MEDICAMENTOS, DOMISSANITÁRIOS E AGROTÓXICOS NA POPULAÇÃO DE JURAMENTO-MG
}

\author{
Ronilson Ferreira Freitas \\ Acadêmico do Curso de Farmácia das Faculdades Integradas Pitágoras de Montes Claros \\ Vanessa de Andrade Royo \\ Doutora em Produtos Naturais e Sintéticos pela USP \\ Docente do curso de Farmácia das Faculdades Integradas Pitágoras de Montes Claros \\ Paula Maria Soares de Moura \\ Mestre em Ginecologia, Obstetrícia e Mastologia pela UNESP \\ Docente das Faculdades Integradas Pitágoras de Montes Claros
}

Recebido em: 18/04/12

Aprovado em: 07/05/12

\begin{abstract}
RESUMO
A intoxicação é a manifestação dos efeitos tóxicos. É um processo patológico causado por substâncias químicas endógenas ou exógenas e caracterizado por desequilíbrio fisiológico, em consequência das alterações bioquímicas no organismo (BATISTUZZO et al, 2008). Esse processo é evidenciado por sinais e sintomas ou mediante exames laboratoriais (CAMPOLINA et al, 2001). Diante deste pressuposto, o objetivo deste trabalho foi avaliar o risco de intoxicações por medicamentos, domissanitários e agrotóxicos na população de Juramento-MG. Quanto à metodologia, trata-se de uma pesquisa de natureza quantitativa, com procedimentos bibliográficos, de campo e objetivos exploratórios. Para a coleta de dados, foram aplicados 121 questionários, constituídos de 33 questões objetivas, cuja população foi escolhida aleatoriamente. Os resultados demonstraram que: o índice de intoxicações na cidade é de 33,1\%, um índice não muito alto, entretanto, observando os riscos que as intoxicações trazem para o organismo humano, esse número é significativo. Foi possível observar também que essa população apresenta pouco conhecimento dos riscos que correm em relação às intoxicações, sendo possível concluir que normas da prevenção de intoxicações transitam pela boa educação sanitária, pela responsabilidade na utilização de substâncias tóxicas, favorecendo a aprendizagem para uma vida saudável. Observa-se a necessidade de campanhas educativas para ampliar o conhecimento e o cuidado da população em relação aos riscos que correm com produtos que são altamente tóxicos.
\end{abstract}

Palavras-chave: Intoxicações. Medicamentos. Domissanitários. Agrotóxicos.

\section{RISK ASSESSMENT OF POISONINGS DUE TO DRUGS, HOUSEHOLD CLEANING AND PESTICIDES IN THE POPULATION OF JURAMENTO-MG}




\begin{abstract}
Poisoning is the manifestation of toxic effects. It is a pathological process caused by endogenous or exogenous chemicals and characterized by physiological imbalance as a result of biochemical changes in the body (BATISTUZZO et al, 2008). This process is evidenced by signs and symptoms or by laboratory tests (CAMPOLINA et al, 2001). Given this assumption, the objective of this study was to evaluate the risk of poisoning by drugs, household cleaning and pesticides in the population of Juramento-MG. Regarding methodology, it is a quantitative survey, with bibliographic procedures, field and exploratory objectives. For data collection, 121 questionnaires were applied, consisting of 33 objective questions, whose population was chosen randomly. The results showed that: the rate of poisoning in the city is $33.1 \%$, a rate not too high, however, noting that risks poisoning bring to the human body, this number is significant. It was also observed that this population has little knowledge of this risks they face in relation to poisoning, it is possible to conclude that rules prevent poisonings transiting through good health education, the responsibility for the use of toxic substances, enabling them to learn for a healthy life. There is a need for educational campaigns to increase knowledge and care of the population in relation to the risks they face with products that are higly toxic.
\end{abstract}

Keywords: Poison. Medicines. Household cleaning. Pesticides.

\title{
1 INTRODUÇÃO
}

A intoxicação, segundo Villas Boas et al (2009), é caracterizada como um conjunto de sinais e sintomas que demonstram o desequilíbrio orgânico promovido pela ação de uma substância química, ou seja, é a manifestação dos efeitos tóxicos que são caracterizados por desequilíbrio fisiológico, em decorrência das alterações bioquímicas que ocorrem no organismo. Processo que pode ser evidenciado até mesmo por realização de exames laboratoriais (CAMPOLINA et al, 2001).

Dentre as intoxicações, destaca-se que os maiores índices são causados por medicamentos, produtos domissanitários e agrotóxicos. A toxicologia é a área responsável por estudar os efeitos nocivos produzidos pela interação de substâncias químicas com o organismo (OGA; CAMARGO; BATISTUZZO, 2008).

Segundo estudos realizados, para que o tratamento de intoxicações tenha resultados positivos, faz-se necessária a identificação da substância responsável pela intoxicação e conhecimento específico de suas características químicas, metabólicas e excreção, bem como a fisiopatologia do envenenamento e inativação dos efeitos causados por estas substâncias (ALVAREZ; ELENA; HERNANDEZ, 2000).

No Brasil, pesquisadores afirmam, segundo Santana Ral et al (2011), que os casos de envenenamentos ocasionados da falta de orientação da população sobre a indicação e os produtos químicos disponíveis no mercado vêm se multiplicando desde do século passado na década de noventa. 
De acordo com dados fornecidos pelo Sistema Nacional de Informações Tóxico-Farmacológicas (Sinitox) da Fiocruz (2010), mais de 100 mil casos de intoxicação humana e quase 500 óbitos são registrados pelos Centros de Informação e Assistência Toxicológica espalhados pelo país. Esses dados apontam que os medicamentos $(30,7 \%)$, animais peçonhentos $(20,1 \%)$ e produtos domissanitários $(11,4 \%)$ são os principais agentes causadores de intoxicações em seres humanos, entretanto, existem outros produtos que provocam ocorrência de intoxicações pelos mesmos, como por exemplo, os agrotóxicos.

Assim é possível observar que o crescimento das intoxicações vem sendo seguidamente destacado pela equipe técnica do Sinitox (Sistema Nacional de Informações Tóxico-Farmacológicas), nas análises de dados encontrados nas publicações anuais de estatísticas de casos de intoxicação e envenenamento no Brasil. Frente a esta situação de intoxicações, este estudo procura ouvir a população da cidade de Juramento-MG e verificar como elas compreendem e vivenciam essa situação. Desta forma, a realização de um trabalho com essa população, que identifique fatores pertencentes ao contexto das intoxicações nessa comunidade, vai favorecer a criação e implementação de medidas voltadas à prevenção desses agravos.

O presente estudo teve como objetivo geral avaliar o risco de intoxicações por medicamentos, domissanitários e agrotóxicos na população de Juramento-MG.

\section{MÉTODOS}

Este estudo foi realizado pelo grupo de acadêmicos e professores do terceiro período do curso de Farmácia das Faculdades Integradas Pitágoras de Montes Claros, do qual os autores fazem parte. Trata-se de uma pesquisa de natureza quantitativa, com procedimentos bibliográficos, de campo e objetivos exploratórios. O cenário da pesquisa foi a população da cidade de Juramento-MG, onde a coleta de dados foi realizada em novembro de 2010. A cidade de Juramento-MG está localizada na Região VI-Noroeste, sua altitude máxima é de 1256 m na Serra do Caturi e a mínima de 642 m no Rio da Prata. A área de Juramento é de 432,52 $\mathrm{Km}^{2}$ e fica a $36 \mathrm{Km}$ de Montes Claros-MG. Sua população estimada em 2010 é de aproximadamente cinco mil habitantes, segundo dados fornecidos no site da Prefeitura Municipal.

Para a coleta de dados, foi utilizado um questionário padronizado, constituído de 33 questões fechadas. Os acadêmicos foram distribuídos entre os bairros da cidade, sendo que cada acadêmico visitou de quatro a seis casas. A amostra foi constituída de 121 pessoas de ambos os sexos, escolhidas aleatoriamente. Os participantes assinaram um termo de consentimento livre e esclarecido autorizando 
sua participação nesta pesquisa. A análise dos dados foi realizada com a ajuda do Software Windows SPSS versão 11.0.

\section{RESULTADOS E DISCUSSÃO}

Os resultados obtidos na pesquisa, cujo objetivo era avaliar o risco de intoxicações por medicamentos, domissanitários e agrotóxicos, foram significativos.

Foram entrevistadas 121 pessoas. Destas, 3,3\% tinham abaixo de 15 anos, $14 \%$ entre 16 e 20 anos, 22,3\% possuiam idade entre 21 e 30 anos, 21,5\% entre 31 e 40 anos, 25,6\% tinham faixa etária entre 41 e 60 anos e 13,2\% possuíam mais de 60 anos, conforme mostra a Tabela 1. E a maioria dos entrevistados eram do sexo feminino $(70,2 \%)$ e os outros $29,8 \%$ eram do sexo masculino.

Tabela 1 - Idade dos entrevistados

\begin{tabular}{lccl}
\hline Faixa Etária & Frequiência & Porcentagem & $\begin{array}{c}\text { Porcentagem } \\
\text { Simples }\end{array}$ \\
\hline$<15$ anos & 4 & $3,3 \%$ & $3,3 \%$ \\
$16-20$ anos & 17 & $14,0 \%$ & $14,0 \%$ \\
$21-30$ anos & 27 & $22,3 \%$ & $22,3 \%$ \\
$31-40$ anos & 26 & $21,5 \%$ & $21,5 \%$ \\
$41-60$ anos & 31 & $25,6 \%$ & $25,5 \%$ \\
$>60$ anos & 16 & $13,2 \%$ & $13,2 \%$ \\
& & & \\
Total & 121 & $100 \%$ & $100 \%$ \\
\hline
\end{tabular}

Fonte: Pesquisa realizada pelos acadêmicos do $3^{\circ}$ período do curso de Farmácia das FIPMoc

No questionário, uma das questões estava relacionada aos casos de intoxicações na família do entrevistado e 66,9\% responderam que nunca houve casos de intoxicação na família e 33,1\% responderam que já houve casos de intoxicação na família, o que significa um percentual bem elevado em relação aos agravos que as intoxicações podem causar no organismo.

Dentre os casos positivos para intoxicações na família dos entrevistados (tabela 2), destaca-se que a faixa etária mais comum de ocorrer intoxicação é abaixo dos 15 anos e entre 41-60 anos, o que é caracterizado em partes na literatura escrita por Villas Boas et al. (2009), que diz que em crianças e adultos acima dos 60 anos é observado o maior índice de intoxicações. 
Tabela 2 - Idade do intoxicado na família do entrevistado

\begin{tabular}{lccc}
\hline Faixa Etária & Frequência & Porcentagem & $\begin{array}{c}\text { Porcentagem } \\
\text { Simples }\end{array}$ \\
\hline$<15$ anos & 14 & $11,6 \%$ & $11,6 \%$ \\
$16-20$ anos & 2 & $1,7 \%$ & $1,7 \%$ \\
$21-30$ anos & 7 & $5,8 \%$ & $5,8 \%$ \\
$31-40$ anos & 5 & $4,1 \%$ & $4,1 \%$ \\
$41-60$ anos & 11 & $9,1 \%$ & $9,1 \%$ \\
$>60$ anos & 1 & $0,8 \%$ & $0,8 \%$ \\
Nunca Houve & 81 & $66,9 \%$ & $66,9 \%$ \\
& & & \\
Total & 121 & $100 \%$ & $100 \%$ \\
\hline
\end{tabular}

Fonte: Pesquisa realizada pelos acadêmicos do $3^{\circ}$ período do curso de Farmácia das FIPMoc

A disponibilidade crescente e a utilização cada vez maior de substâncias químicas, incluindo medicamentos, agrotóxicos e domissanitários, durante as últimas décadas, acarretaram uma elevação de risco à saúde humana provocada pela exposição a estes agentes. (LOPES et al, 2006). Segundo a Organização Mundial de Saúde (OMS), dezenas de milhares de substâncias produzidas pelo homem estão em uso no mundo, e cerca de mil a duas mil novas substâncias químicas entram no mercado a cada ano. Todo indivíduo está exposto diariamente, por alguns minutos, a substâncias químicas em doses subtóxicas, por meio de contaminação medicamentosa, por agrotóxico, por produtos domissanitários, entre outros. (LOPES et al, 2006).

De acordo com a pesquisa realizada, foi possível perceber que os produtos que mais causam intoxicações na população de Juramento foram os domissanitários (11,6\%), em segundo lugar, os medicamentos $(9,1 \%)$ e em terceiro lugar, os agrotóxicos $(2,5 \%)$. O índice de intoxicação por domissanitários foi muito alto, ultrapassando a média nacional informada pela Fiocruz (2010), entretanto, os dados obtidos na pesquisa realizada em Juramento contradizem os dados disponibilizados pelo Sistema Nacional de Informações Tóxico-Farmacológicas (Sinitox) da Fiocruz (2010) que diz que os medicamentos estão em primeiro lugar nos produtos que mais causam intoxicação, sendo que neste estudo realizado, observa-se que o maior causador de intoxicações nessa população foram os produtos domissanitários (TAB. 3). 
Tabela 3 - Produtos que causaram a intoxicação

\begin{tabular}{lrcc}
\hline Produto & Frequência & Porcentagem & $\begin{array}{c}\text { Porcentagem } \\
\text { Simples }\end{array}$ \\
\hline Domissanitários & 14 & $11,6 \%$ & $11,6 \%$ \\
Medicamentos & 11 & $9,1 \%$ & $9,1 \%$ \\
Agrotóxicos & 3 & $2,5 \%$ & $2,5 \%$ \\
Outros & 12 & $9,9 \%$ & $9,9 \%$ \\
Nunca Houve & 81 & $66,9 \%$ & $66,9 \%$ \\
Total & 121 & $100 \%$ & $100 \%$ \\
\hline
\end{tabular}

Fonte: Pesquisa realizada pelos acadêmicos do $3^{\circ}$ período do curso de Farmácia das FIPMoc

Segundo Villas Boas et al (2009), os prováveis fatores que determinam os acidentes que causam intoxicação são: produto ao alcance de crianças, armazenamento em frasco inadequado causando confusão com refrigerantes, água, alimento medicamentos e cosméticos; ocorrência do acidente durante a utilização do produto, uso indevido do produto, entre outros. E os resultados obtidos não foram muito diferentes, em que acidente individual e uso indevido tiveram um percentual de 9,9\%, erro de administração 3,3\% e as outras circunstâncias citadas tiveram um percentual de 76,9\%, um resultado bastante significativo. (TAB. 4).

Tabela 4 - Produtos que causaram a intoxicação

\begin{tabular}{lccc}
\hline Circunstância & Frequência & Porcentagem & $\begin{array}{c}\text { Porcentagem } \\
\text { Simples }\end{array}$ \\
\hline Acidente Individual & 12 & $9,9 \%$ & $9,9 \%$ \\
Erro de Administração & 4 & $3,3 \%$ & $3,3 \%$ \\
Uso indevido & 12 & $9,9 \%$ & $9,9 \%$ \\
Outros & 93 & $76,9 \%$ & $76,9 \%$ \\
Total & & & \\
\hline
\end{tabular}

Fonte: Pesquisa realizada pelos acadêmicos do $3^{\circ}$ período do curso de Farmácia das FIPMoc.

Em casos de intoxicação, dentre as medidas preventivas, destaca-se levar a vítima imediatamente para o hospital, onde a maioria das pessoas intoxicadas recebem alta quando atendidas de forma rápida e eficaz. O primeiro atendimento ao paciente intoxicado deve visar o restabelecimento das funções vitais e só depois é que se deve partir para tratamentos específicos (CEATOX-IJF, 2010).

Entretanto, nem todos os entrevistados que disseram que já ocorreram casos de intoxicação na família realizam os procedimentos mencionados anteriormente, sendo que 22,3\% dos entrevistados responderam que levaram a vítima imediatamente para o hospital, 1,7\% provocaram vômitos e fizeram 
com que o paciente ingerisse leite. Segundo Guerra et al (2006), nunca deve-se dar para a pessoa intoxicada ovos crus, sal, vinagre ou sucos de frutas cítricas e leite para induzir vômitos ou neutralizar o veneno sem recomendação médica, pois estes procedimentos podem ser fatais para a vitima e 7,4\% dizem ter realizado outros procedimentos não informados aqui.

Tabela 5 - Procedimentos realizados com a vitima

\begin{tabular}{lccc}
\hline Procedimentos & Frequência & Porcentagem & Porcentagem \\
\hline $\begin{array}{c}\text { Levou o paciente } \\
\text { o mais rápido } \\
\text { para o hospital }\end{array}$ & 27 & $22,3 \%$ & $22,3 \%$ \\
$\begin{array}{c}\text { Provocou vômitos } \\
\text { do paciente }\end{array}$ & 2 & $1,7 \%$ & $1,7 \%$ \\
$\begin{array}{c}\text { Fez com que o } \\
\text { paciente ingerisse } \\
\text { leite }\end{array}$ & 2 & $1,7 \%$ & $1,7 \%$ \\
$\quad \begin{array}{l}\text { Outros } \\
\text { Nunca presenciou } \\
\text { caso de intoxicação }\end{array}$ & 81 & $66,9 \%$ & $66,9 \%$ \\
\begin{tabular}{l} 
Total \\
\hline
\end{tabular} & 9 & $7,4 \%$ & $7,4 \%$ \\
\hline
\end{tabular}

Fonte: Pesquisa realizada pelos acadêmicos do $3^{\circ}$ período do curso de Farmácia das FIPMoc.

\section{CONCLUSÃO}

As intoxicações são um problema muito comum que atinge muitos brasileiros diariamente, e os resultados obtidos nesta pesquisa realizada na população de Juramento-MG estão dentro da realidade do Brasil, apesar de que, se compararmos os números, os resultados podem até ser menores, entretanto, se partirmos para analisar os danos que os efeitos tóxicos causam ao organismo do indivíduo, este número se torna bem significativo.

Foi possível observar também a carência de informações que essa população possui em relação à aquisição, armazenamento e utilização de produtos altamente tóxicos, o que contribui para os resultados obtidos.

Sendo assim, conclui-se que normas da prevenção de intoxicações transitam pela boa educação sanitária, pela responsabilidade na utilização de substâncias tóxicas, favorecendo a aprendizagem para uma vida saudável, e observa-se a necessidade de campanhas educativas nessa população, com o 
objetivo de ampliar o conhecimento e cuidado da população de Juramento em relação aos riscos que eles correm com estes produtos.

\section{REFERÊNCIAS}

ALVAREZ, E. P. et al. Sistematicamente utilizados no Centro Nacional de Toxicologia. Emergências toxicológicas. Revista Cubana de Medicina Militar, Havana, v. 29, n. 1, jan./abr. 2000.

ANDRADE FILHO, A.; CAMPOLINA, D.; DIAS, M. B. Toxicologia na prática. Belo Horizonte: Folium, 2001.

BATISTUZZO, J. A. O.; CAMARGO, M. A.; OGA, S. Fundamentos de toxicologia. São Paulo: Atheneu, 2008.

CENTRO DE ASSISTÊNCIA TOXICOLÓGICA-CEATOX. Cuidado com embalagens previne acidentes. Disponível em: http://www.ijf.ce.gov.br. Acesso em: 15 ago. 2010.

DELAMARE, J. Dicionário Médico Andrei. São Paulo: Ed. Andrei, 1997.

GUERRA, L. R. et al. Intoxicação por domissanitário. Conduta médica, Rio de Janeiro, v. 7, n. 27 , 2006.

INTOXICAÇÃO MEDICAMENTOSAS. Disponível em: <http://www.fiocruz.br/sinitox_novo/cgi/cgilua.exe/sys/start.htm?tpl=home> Acesso em: 16 ago. 2010.

LOPES, A. C. Fundamentos de toxicologia clínica. São Paulo: Atheneu, 2006.

OGA, S. et al. Fundamentos de toxicologia. 3. ed. São Paulo: Atheneu, 2008.

OPAS/OMS. Manual de Vigilância da Saúde de populações expostas à agrotóxicos . Brasília/DF, 1996.

PREFEITURA MUNICIPAL DE JURAMENTO-MG. População estimada em 2010. Disponível em: www.juramento.mg.gov.br Acesso em: 21 nov. 2010.

SANTANA, R. A. L. de; BOCHNER, R.; GUIMARAES, M. C. S. Sistema nacional de informações tóxico-farmacológicas: o desafio da padronização dos dados. Ciência e saúde coletiva, Rio de Janeiro, v. 16, supl. 1, 2011.

SISTEMA NACIONAL DE INFORMAÇÃO TÓXICO-FARMACOLOGICOS. Novos dados sobre intoxicação humana. Disponível em: http//www.fiocruz.br/sinitox/envenenamento domestico. Acesso em: 13 ago. 2010.

VILLAS BOAS, M. H. et al. Análise dos dados dos Centros de Controle de Intoxicação do Rio de Janeiro, Brasil, como subsidio as ações de saúde pública. Cadernos de Saúde Pública, Rio de Janeiro. v. 25, n. 2, p. 401-408, fev. 2009. 